\title{
Learning to stream and streaming to learn
}

\author{
Stevie Prickett \\ University of Cumbria
}

Keywords: performance; creative technologies; streaming; Covid-19.

\section{The problem}

How do you assess productions on Performing Arts programmes if there is no audience? This was a significant problem facing me and my colleagues within the Performing Arts department when returning to a blended learning model at the beginning of the 20/21 academic year.

Planning had taken place over the summer for the delivery of skills or seminar-based modules but the uncertainty over government guidance meant we were creating multiple, increasingly complex contingencies for solutions that might never be needed.

The specific problem was that we had level 5 and 6 students on four performing arts programmes who were due to undertake performance projects with guest directors and choreographers. The modules were designed to assess the students' ability to perform for an audience. In addition, students had to engage in group creative practice to demonstrate integration of practical skills in their live performance. The problem was, therefore, how to create a model of delivery and assessment which allowed the students to develop the skills necessary to meet module learning outcomes through live performance with no audience present.

\section{The response}

An agreed strategy to protect the student learning experience was to explore the possibility of live streaming performances. This necessitated a skills exchange and new collaborative 
partnership between staff from Performing Arts, Film and TV and Technical Services. On being tasked with leading the project across the year, using an action research model, my primary concerns were:

- Ensuring the students still had a live performance experience.

- Maintaining a model in which work was assessed live not through filmed outcomes.

- Working with existing technologies and equipment within the Institute of the Arts to develop a new model of engaging with audiences.

- Taking learning from the performance modules to develop and enhance online teaching approaches and improve student engagement.

- Creating a model of delivery and assessment which would require minimal revision in response to changing government guidance.

Failing to engage with a new form of delivery would compromise the student experience, deny them live performance experience, and fail to provide opportunities to test and develop the skills they had been progressing during their time on their programmes. In addition, the project had the potential to develop a new range of skills and experiences responsive to and suitable for the emerging industry environment. It would also allow student work to reach a more geographically diverse audience and develop an artistic archive of the work across the year. It also had the potential for cross discipline collaboration and learning for both staff and students.

Initial conversations revolved around the resourcing of equipment, space, time, virtual platforms and staffing. Four performances across the year were highlighted as case studies which could each test a different engagement with streaming. In choosing these productions, I had to consider where the 'liveness' existed in each of the different models of working. It was also possible to increase the creative and technical complexity from one project to the next in response to the learning. The performances were:

- Dance production - streamed live with a single camera feed in auditorium. Students assessed on live performance.

- Musical theatre production - filmed and edited for broadcast. Students assessed on performance during filming. 
- Drama production - filmed as live with multi-camera set up in multiple sets.

Students assessed on live performance during filming.

- Acting performance - devised show streamed live from a multi-camera set-up.

Students assessed on performance for camera in the live stream.

The first production was devised for a traditional theatre set up, but from then on the works were created with the understanding they were never meant to be performed to an audience in situ.

Issues arising included: maintaining social distancing guidance during rehearsal and performance, no sharing of props, guidance on the setting up and operation of technical equipment, and a second national lockdown. This second lockdown became easier to negotiate, as I could adapt projects designed for remote viewing rather than needing to be reactive at short notice.

The project was beneficial to both staff and students. Staff were able to collaborate with colleagues in new creative ways and were also upskilled in the use of technology and equipment. Students had access to new models of performance which they would not have covered in a traditional academic year. Staff and students collaborated to find creative solutions to technical and performance demands. For me, it also raised the question of notions of 'liveness'.

Student feedback on their learning has been positive. There was initially a sense of compromise when they were performing to an empty theatre and a feeling that they were not getting the full live performance experience. However, students became enthused when working on a production when streaming was always the intended outcome and felt they were learning new skills which were relevant and applicable to a changing professional workplace.

\section{Recommendations}

As the project progressed, it became apparent that a new learning environment was being created. Staff members were gaining experience of new models of streamed performance work, software such as OBS (Open Broadcaster Software, a free and open source software for video recording and live streaming), and modes of engagement that are 
commonplace on streaming platforms such as YouTube, Discord and Twitch. Students were utilising and learning new performance and technical skills in a new medium.

Designing the project for remote/virtual audiences and for a different media reduced the students' perception of online delivery as lesser than face-to-face. This in turn improved student engagement and the value they placed on the learning they were undertaking. The project did not try to transplant an existing model to a new medium, but designed a new model tailored to the possibilities and restrictions of live streaming. This supports the idea that we 'should aim for hybrid learning, designing for optimum synergy between online learning and teaching interventions' (Daniel, 2016, no pagination). This model also helped to minimise complex contingencies for delivery.

Engagement with these new technologies does not require the rejection of old systems, but finding ways to integrate the two. There is then the possibility of being live and virtual simultaneously for both us and our students. This model of working brings its own challenges requiring further exploration, especially around how learning is experienced and embedded.

What this project has highlighted is that online delivery works better when we design and tailor according to the medium. Students are more engaged as the learning is embedded and enhanced by the medium and is not a substitute for what they could get in a face-toface environment. It also allows the online delivery to support the face-to-face learning and vice versa, an approach supported by pre-Covid research which found that 'improving approaches to learning and teaching online leads to enhancements in face-to-face learning environments' (Peacock et al., 2012, p.1278).

In addition to sharing knowledge and skills, continued collaboration with colleagues from other departments allows discipline specialists to develop new and innovative models of delivery together. Individual projects can then be a valuable learning opportunity for students from different programmes.

Moving forward, I would suggest understanding what each medium is capable of to equip ourselves with a wider range of skills and resources to enhance delivery, exploring which format is most suitable when, and shaping delivery so both online and face-to-face feel apposite and synchronous. By designing in this way, we are asking 'not which is the best 
method overall, but: What are the most appropriate conditions for using face-to-face, blended or fully online learning?' (Bates, 2015, p.370).

The learning we have done this year has given us discipline-specific knowledge and demonstrates a need for flexibility and responsiveness in the design of online learning environments.

\section{References}

Bates, A.W. (2015) Teaching in a digital age: guidelines for designing teaching and learning. Available at: http://opentextbc.ca/teachinginadigitalage/ (Accessed: 3 June 2021).

Daniel, J. (2016). Making sense of blended learning: treasuring an older tradition or finding a better future. Available at: https://teachonline.ca/tools-trends/blended-learningsuccessful-design-delivery-and-student-engagement/making-sense-blendedlearning-treasuring-older-tradition-or-finding-better-future (Accessed: 1 October 2021).

Peacock, S., Murray, S., Dean, J., Brown, D., Girdler, S. and Mastrominico, B. (2012) 'Exploring tutor and student experiences in online synchronous learning environments in the performing arts', Creative Education, 3, pp.1269-1280.

Available at: http://dx.doi.org/10.4236/ce.2012.37186 (Accessed: 1 October 2021).

\section{Author details}

Stevie Prickett has worked as a theatre maker and performer for over 20 years and is currently Lecturer in Dance and Physical Theatre at the University of Cumbria. His research interests include interdisciplinary practice and engaging with creative technologies in the creation and teaching of Performing Arts. 